\title{
Total Synthesis of Debromoflustramine B via Biomimetic Alkylative Cyclization
}

Gan Hup Tan, Xiuwen Zhu, and A. Ganesan*

Supporting information.

Synthesis of carbamate 1. Tryptamine $(2.40 \mathrm{~g}, 15 \mathrm{mmol})$ was dissolved in a water (30 $\mathrm{mL})$ and dichloromethane $(30 \mathrm{~mL})$ mixture and treated with $\mathrm{NaCl}(3.75 \mathrm{~g})$ and $\mathrm{NaHCO}_{3}$ $(1.28 \mathrm{~g}, 22.5 \mathrm{mmol})$ at $0 \mathrm{C}$. Ethyl chloroformate $(1.65 \mathrm{~mL}, 22.5 \mathrm{mmol})$ was added dropwise via a syringe, and the reaction stirred for $3 \mathrm{~h}$ (monitored by TLC, 1:1 EtOAc:Hex). The organic layer was then separated and the aqueous layer washed with dichloromethane (2 $20 \mathrm{~mL}$ ). The combined organic extract was washed with brine (2 $20 \mathrm{~mL}$ ), dried, concentrated and purified by column chromatography (silica, 1:2 EtOAc:Hex) to yield 1 as a pale yellow viscous oil (2.94 g, $12.7 \mathrm{mmol}, 84 \%)$. IR 3313, $1688 \mathrm{~cm}^{-1} ;{ }^{1} \mathrm{H}$ NMR (300 MHz, $\left.\mathrm{CDCl}_{3}\right) \quad 8.07$ (1H, br s, CNHCH), $7.61(1 \mathrm{H}, \mathrm{d}, \mathrm{J} 8.1$ $\mathrm{Hz}, \mathrm{CCHCH}), 7.37$ (1H, d, J $7.4 \mathrm{~Hz}, \mathrm{CCHCH}), 7.21(1 \mathrm{H}, \mathrm{t}, \mathrm{J} 7.4 \mathrm{~Hz}, \mathrm{CHCHCH}), 7.13$ $(1 \mathrm{H}, \mathrm{t}, \mathrm{J} 6.5 \mathrm{~Hz}, \mathrm{CHCHCH}), 7.04(1 \mathrm{H}, \mathrm{s}, \mathrm{NHCH}), 4.70(1 \mathrm{H}, \mathrm{br} \mathrm{s}, \mathrm{NHC}=\mathrm{O}), 4.11(2 \mathrm{H}, \mathrm{q}$, J $\left.7.4 \mathrm{~Hz}, \mathrm{CH}_{2} \mathrm{CH}_{3}\right), 3.51\left(2 \mathrm{H}, \mathrm{t}, \mathrm{NHCH}_{2} \mathrm{CH}_{2}\right), 2.97\left(2 \mathrm{H}, \mathrm{t}, \mathrm{J} 6.5 \mathrm{~Hz}, \mathrm{NHCH}_{2} \mathrm{CH}_{2}\right), 1.23$ $\left(3 \mathrm{H}, \mathrm{t}, \mathrm{J} 7.4 \mathrm{~Hz}, \mathrm{CH}_{2} \mathrm{CH}_{3}\right) ;{ }^{13} \mathrm{C} \mathrm{NMR}\left(75.5 \mathrm{MHz}, \mathrm{CDCl}_{3}\right) \quad 156.8(\mathbf{C}), 136.4(\mathbf{C}), 127.3$ (C), $122.2(\mathbf{C H}), 122.1(\mathbf{C H}), 119.2(\mathbf{C H}), 118.7(\mathbf{C H}), 112.1(\mathbf{C}), 111.2(\mathbf{C H}), 60.8$ $\left(\mathbf{C H}_{2}\right), 41.2\left(\mathbf{C H}_{2}\right), 25.8\left(\mathbf{C H}_{2}\right), 14.7\left(\mathbf{C H}_{3}\right)$; ESI+ MS m/z $233\left([\mathrm{M}+\mathrm{H}]^{+}, 23 \%\right), 255(14)$, 487 (50). Spectroscopic data was identical to that reported in the literature.

Synthesis of hexahydropyrrolo[2,3-b]indoline 3. Carbamate $2(0.200 \mathrm{~g}, 0.86 \mathrm{mmol})$ was added to a mixture of zinc triflate $(0.313 \mathrm{~g}, 0.86 \mathrm{mmol})$, tetra-butyl ammonium iodide $(0.635 \mathrm{~g}, 1.72 \mathrm{mmol}), N, N$-diisopropylethylamine $(0.330 \mathrm{~mL}, 1.89 \mathrm{mmol})$ and toluene $(5 \mathrm{~mL})$ under a nitrogen atmosphere at room temperature. The mixture was stirred for $15 \mathrm{mins}$ before dropwise addition of prenyl bromide $(0.397 \mathrm{~mL}, 3.44 \mathrm{mmol})$. The reaction mixture was stirred for $3 \mathrm{~h}$ and monitored by TLC (2:1). Upon completion, the reaction was quenched with saturated $\mathrm{NH}_{4} \mathrm{Cl}(5 \mathrm{~mL})$, diluted with $\mathrm{H}_{2} \mathrm{O}(5 \mathrm{~mL})$ and extracted with ether (2 $5 \mathrm{~mL})$. The resulting organic layer was washed with water, dried, concentrated and purified by column chromatography (silica, 1:5 EtOAc:Hex), to yield 3 as a pale yellow oil $(0.220 \mathrm{~g}, 0.60 \mathrm{mmol}, 70 \%)$. IR $1693,1604,1486 \mathrm{~cm}^{-1} ;{ }^{1} \mathrm{H}$ 
NMR (400 MHz, CDCl $) 7.06$ (1H, dt, J 7.8, 1.3 Hz, CHCHCH), 6.97 (1H, d, J 7.0 Hz, NCCHCH), 6.65 (1H, t, J 7.4 Hz, CHCHCH), 6.36 (1H, d, J 7.4 Hz, CCHCH), $5.35(1 \mathrm{H}$, s, NCHN), $5.16\left(1 \mathrm{H}, \mathrm{t}, \mathrm{J} 7.0 \mathrm{~Hz}, \mathrm{CHCH}_{2} \mathrm{~N}\right), 5.06\left(1 \mathrm{H}, \mathrm{t}, \mathrm{J} 7.0 \mathrm{~Hz}, \mathrm{CCH}_{2} \mathrm{CH}\right), 4.15(2 \mathrm{H}$, q, J $\left.6.8 \mathrm{~Hz}, \mathrm{CH}_{3} \mathrm{CH}_{2} \mathrm{O}\right), 3.96\left(2 \mathrm{H}\right.$, d, J $\left.7.0 \mathrm{~Hz}, \mathrm{NCH}_{2} \mathrm{CH}\right), 3.70-3.78(1 \mathrm{H}, \mathrm{m}$, $\left.\mathrm{NCHHCH}_{2}\right), 3.05\left(1 \mathrm{H}, \mathrm{dt}, \mathrm{J} 8.4,9.5 \mathrm{~Hz}, \mathrm{NCHHCH}_{2}\right), 2.38\left(2 \mathrm{H}, \mathrm{d}, \mathrm{J} 7.5 \mathrm{~Hz}, \mathrm{CCH}_{2} \mathrm{CH}\right)$, $2.02\left(2 \mathrm{H}, \mathrm{dd}, \mathrm{J} 6.0,8.3 \mathrm{~Hz}, \mathrm{NCH}_{2} \mathrm{CH}_{2}\right), 1.74\left(3 \mathrm{H}, \mathrm{s}, \mathrm{CHCCH}_{3}\right), 1.68(6 \mathrm{H}, \mathrm{s}$, $\left.\mathrm{CHC}\left(\mathrm{CH}_{3}\right)_{2}\right), 1.55\left(3 \mathrm{H}, \mathrm{s}, \mathrm{CHCCH}_{3}\right), 1.26\left(3 \mathrm{H}, \mathrm{t}, \mathrm{J} 6.8 \mathrm{~Hz}, \mathrm{OCH}_{2} \mathrm{CH}_{3}\right) ;{ }^{13} \mathrm{C}$ NMR $(100$ $\left.\mathrm{MHz}, \mathrm{CDCl}_{3}\right) \quad 150.2(\mathbf{C}), 134.6(\mathbf{C}), 133.2(\mathbf{C}), 128.4(\mathbf{C H}), 124.3(\mathbf{C H}), 122.7(\mathbf{C H})$, $121.4(\mathbf{C H}), 119.5(\mathbf{C H}), 117.0(\mathbf{C H}), 106.4(\mathbf{C H}), 84.4(\mathbf{C H}), 61.0(\mathbf{C H}), 56.2(\mathbf{C}), 45.4$ $\left(\mathbf{C H}_{2}\right), 44.2\left(\mathbf{C H}_{2}\right), 37.0\left(\mathbf{C H}_{2}\right), 36.9\left(\mathrm{CH}_{2}\right), 36.8\left(\mathrm{CH}_{2}\right), 25.9\left(\mathrm{CH}_{3}\right), 25.7\left(\mathrm{CH}_{3}\right), 18.0$ $\left(\mathrm{CH}_{3}\right), 14.7\left(\mathrm{CH}_{3}\right) ; \mathrm{ESI}+\mathrm{MS} m / z 759.3\left[2 \mathrm{M}+\mathrm{Na}^{+}, 95 \%\right]$.

Synthesis of debromoflustramine B. To carbamate $3(0.368 \mathrm{~g}, 1.00 \mathrm{mmol})$ in toluene (5 $\mathrm{mL}$ ) was added Red-Al (10.0 mmol, $0.195 \mathrm{~mL}$ of Aldrich $65+\mathrm{wt} . \%$ solution in toluene). The reaction mixture was refluxed under nitrogen for $24 \mathrm{~h}$ until completion, as monitored by TLC (1:2 EtOAc:Hex). The reaction mixture was then treated with EtOAc $(200 \mathrm{~mL})$ and washed with water $(50 \mathrm{~mL})$, brine $(220 \mathrm{~mL})$, dried and concentrated. Purification by column chromatography (silica, EtOAc) afforded debromoflustramine B as a pale yellow oil (0.300 g, $0.97 \mathrm{mmol}, 96 \%)$. IR 1670, 1601, $1485 \mathrm{~cm}^{-1}$; ${ }^{1} \mathrm{H}$ NMR (400 MHz, $\left.\mathrm{CDCl}_{3}\right) 7.04(1 \mathrm{H}, \mathrm{td}, \mathrm{J} 8.0,1.5 \mathrm{~Hz}, \mathrm{CHCHCH}), 6.97(1 \mathrm{H}, \mathrm{dd}, \mathrm{J}$ 7.3, $1.3 \mathrm{~Hz}, \mathrm{NCCHCH})$, $6.64(1 \mathrm{H}, \mathrm{td}, \mathrm{J} 7.5,1.0 \mathrm{~Hz}, \mathrm{CHCHCH}), 6.41(1 \mathrm{H}, \mathrm{br} \mathrm{d}, \mathrm{J} 7.8 \mathrm{~Hz}, \mathrm{CCHCH}), 5.17(1 \mathrm{H}, \mathrm{br}$ t, J $\left.4.4 \mathrm{~Hz}, \mathrm{CHCH}_{2} \mathrm{~N}\right), 4.97\left(1 \mathrm{H}\right.$, br t, J $\left.5.6 \mathrm{~Hz}, \mathrm{CCH}_{2} \mathrm{CH}\right), 4.26(1 \mathrm{H}, \mathrm{s}, \mathrm{NCHN}) ; 3.92(1 \mathrm{H}$, dd, J 16.0, 5.8 Hz, NCHHCH), 3.80 (1H, dd, J 16.0, 7.2 Hz, NCHHCH), 2.67 (1H, ddd, J 9.3, 6.5, 3.3. Hz, NCHHCHH), 2.56 (1H, ddd, J 9.0, 5.8, $9.3 \mathrm{~Hz}, \mathrm{NCHHCHH),} 2.48$ (3H, s, $\left.\mathrm{NCH}_{3}\right), 2.42\left(2 \mathrm{H}, \mathrm{d}, \mathrm{J} 7.0 \mathrm{~Hz}, \mathrm{CCH}_{2} \mathrm{CH}\right), 2.00-2.08(1 \mathrm{H}, \mathrm{m}, \mathrm{NCHHCHH}), 1.88-1.93$ $(1 \mathrm{H}, \mathrm{m}, \mathrm{NCHHCHH}), 1.71\left(3 \mathrm{H}, \mathrm{s}, \mathrm{NCH}_{2} \mathrm{CHC}\left(\mathrm{CH}_{3}\right)_{2}\right), 1.70\left(3 \mathrm{H}, \mathrm{s}, \mathrm{CHC}\left(\mathrm{CH}_{3}\right)_{2}\right), 1.65$ $\left(3 \mathrm{H}, \mathrm{s}, \mathrm{CHC}\left(\mathrm{CH}_{3}\right)_{2}\right), 1.58\left(3 \mathrm{H}, \mathrm{s}, \mathrm{NCH}_{2} \mathrm{CHC}\left(\mathrm{CH}_{3}\right)_{2}\right) ;{ }^{13} \mathrm{C} \mathrm{NMR}\left(100 \mathrm{MHz}, \mathrm{CDCl}_{3}\right)$ 150.9 (C), $134.7(\mathbf{C}), 133.0(\mathbf{C}), 132.4(\mathbf{C}), 126.5(\mathbf{C H}), 121.8(\mathbf{C H}), 120.4(\mathbf{C H}), 119.8$ $(\mathbf{C H}), 116.4(\mathbf{C H}), 106.3(\mathbf{C H}), 90.4(\mathbf{C}), 56.1(\mathbf{C}), 51.8\left(\mathbf{C H}_{2}\right), 45.8\left(\mathbf{C H}_{2}\right), 38.0\left(\mathbf{C H}_{2}\right)$, $37.5\left(\mathbf{C H}_{2}\right), 37.0\left(\mathbf{C H}_{3}\right), 24.9\left(\mathrm{CH}_{3}\right), 24.7\left(\mathrm{CH}_{3}\right), 17.1\left(\mathrm{CH}_{3}\right), 17.0\left(\mathrm{CH}_{3}\right)$; ESI+ MS m/z 
$311\left(\left[\mathrm{M}+\mathrm{H}^{+}\right], 100 \%\right), 325$ (30). Spectroscopic data was identical to that reported in the literature. 